\title{
General Cyclic Orthogonal Double Covers of Finite Regular Circulant Graphs
}

\author{
Ramadan El-Shanawany, Hanan Shabana \\ Department of Physics and Engineering Mathematics, Faculty of Electronic Engineering, Menoufiya University, \\ Menouf, Egypt \\ Email: ramadan elshanawany380@yahoo.com, the engineer hanan@yahoo
}

Received 20 January 2014; revised 18 February 2014; accepted 15 March 2014

Copyright (C 2014 by authors and Scientific Research Publishing Inc.

This work is licensed under the Creative Commons Attribution International License (CC BY).

http://creativecommons.org/licenses/by/4.0/

(c) (i) Open Access

\section{Abstract}

An orthogonal double cover (ODC) of a graph $H$ is a collection $\mathcal{G}=\left\{G_{v}: v \in V(H)\right\}$ of $|V(H)|$ subgraphs (pages) of $H$, so that they cover every edge of $H$ twice and the intersection of any two of them contains exactly one edge. An ODC $\mathcal{G}$ of $H$ is cyclic (CODC) if the cyclic group of order $|V(H)|$ is a subgroup of the automorphism group of $\mathcal{G}$. In this paper, we introduce a general orthogonal labelling for CODC of circulant graphs and construct CODC by certain classes of graphs such as complete bipartite graph, the union of the co-cycles graph with a star, the center vertex of which, belongs to the co-cycles graph and graphs that are connected by a one vertex.

\section{Keywords}

Graph Decomposition, Cyclic Orthogonal Double Cover, Automorphism Group, Orthogonal Labelling

\section{Introduction}

All graphs we deal with are undirected, finite and simple. Let $H$ be any regular graph, and let $\mathcal{G}=\left\{G_{0}, G_{1}, \cdots, G_{|V(H)|-1}\right\}$ be a collection of $|V(H)|$ subgraphs (pages) of $H$. The collection $\mathcal{G}$ is an orthogonal double cover (ODC) of $H$ if it has the following properties:

1) Double cover property:

Every edge of $H$ is contained in exactly two of the pages in $\mathcal{G}$.

2) Orthogonality property:

For any two distinct pages $G_{i}$ and $G_{j} \in \mathcal{G},\left|E\left(G_{i}\right) \cap\left(G_{j}\right)\right|=1$, if and only if $i$ and $j$ are adjacent in $H$. 
If all pages $G_{i} \cong G$, for all $i \in\{0,1, \cdots,|V(H)|-1\}$, then $\mathcal{G}$ is an ODC of $H$ by $G$. An automorphism of an ODC $\mathcal{G}=\left\{G_{0}, G_{1}, \cdots, G_{|V(H)|-1}\right\}$ of $H$ is a permutation $\pi: V(H) \rightarrow V(H)$, such that $\left\{\pi\left(G_{0}\right), \pi\left(G_{1}\right) \cdots, \pi\left(G_{|V(H)|-1}\right)\right\}$, where for $i \in\{0,1, \cdots,|V(H)|-1\}, \pi\left(G_{i}\right)$ is a subgraph of $H$ with $V\left(\pi\left(G_{i}\right)\right)=\left\{\pi(v): v \in V\left(G_{i}\right)\right\}$, and $E\left(\pi\left(G_{i}\right)\right)=\left\{\pi(x) \pi(y): x y \in E\left(G_{i}\right)\right\}$. According to the obvious properties of ODCs by a graph $G$, the underlying graph $H$ has to be $|E(G)|$-regular. This concept is a generalization of the definitions of an ODC of complete graphs and complete bipartite graphs, which has been studied extensively [1]-[2]. El-Shanawny et al. studied extensively the ODC of complete bipartite graphs; see [3]-[6]. An effective method to construct ODCs in the above cases was based on the idea of translate a given subgraph $G$ by a group acting on $V(H)$. If the cyclic group of order $|V(H)|$ is a subgroup of the automorphism group of $\mathcal{G}$ (the set of all automorphisms of $\mathcal{G}$ ), then an ODC $\mathcal{G}$ of $H$ is cyclic (CODC). Therefore, the circulant graph is of special interest. In [7], Scapellato et al. offers some insights on the case on ODC of Cayley graphs on cyclic groups. In [8], Hartmann and Schumacher proved the following: 1) Let $H$ be a 2-regular graph. There exists an ODC of $H$ by $2 K_{2}$ with three exceptions for $H: C_{3}, C_{4}$ and $2 C_{3}$, 2) Let $H$ be a 3-regular graph containing a 1 -factor and without a component isomorphic to $K_{4}$. There exists an ODC of $H$ by $P_{4}, 3$ ) Let $H$ be a 3-regular graph containing a 1-factor and $|V(H)| \geq 24$. There exists an ODC of $H$ by $P_{3}+K_{2}$. In [9], Sampathkumar et al. introduced a special kind of orthogonal labelling called orthogonal $\sigma$-labelling, and they found it for some caterpillars of diameters 4. In [7], Scapellato et al. studied the ODC of Cayley graphs and proved the following: 1 ) All 3-regular Cayley graphs, except $K_{4}$, have ODCs by $P_{4}$, 2) All 3-regular Cayley graphs on Abelian groups, except $K_{4}$, have ODCs by $P_{3}+K_{2}$,

3) All 3-regular Cayley graphs on Abelian groups, except $K_{4}$ and the 3 -prism (Cartesian product of $C_{3}$ and $K_{2}$ ), have ODCs by $3 K_{2}$. In [10], Sampathkumar et al. completely settled the existence problem of CODCs of 4-regular circulant graphs.

The above results on ODCs of graphs with lower degrees motivate us to consider CODCs of graphs with higher degrees. In [11], El-Shanawny et al. deal with cayley graphs on abelian groups and proved the existence of ODCs of cayley graphs by several classes of graphs. Here we are concerned with CODCs of circulant graphs of finite degrees higher than 4 . The paper is organized as follows, Section 1.1 describes the method that can be used throughout. Section-2 constructs CODCs of circulant graphs of finite degrees higher than 4 by certain graph classes. Section 3 offers the general CODCs of circulant graphs.

Definition 1. For a sequence $\left\{d_{1}, d_{2}, \cdots, d_{k}\right\}$ of positive integers with $1 \leq d_{1} \leq d_{2} \leq \cdots \leq d_{k} \leq\lfloor n / 2\rfloor$, the circulant graph $\operatorname{Circ}\left(n ;\left\{d_{1}, d_{2}, \cdots, d_{k}\right\}\right)$, has vertex set $\mathbb{Z}_{n}=\{0,1, \cdots, n-1\}$; two vertices $v_{1}$ and $v_{2}$ are adjacent, if and only if $v_{1}-v_{2} \equiv \pm d_{i}(\bmod n)$, for some $i, i \in\{1,2, \cdots, k\}$.

For an edge $\left\{v_{1}, v_{2}\right\}$ in $\operatorname{Circ}\left(n ;\left\{d_{1}, d_{2}, \cdots, d_{k}\right\}\right)$, the length of $\left\{v_{1}, v_{2}\right\}$ is $\min \left\{\left|v_{1}-v_{2}\right|, n-\left|v_{1}-v_{2}\right|\right\}$. Given two edges $e_{1}=\left\{u_{1}, u_{2}\right\}$ and $e_{2}=\left\{v_{1}, v_{2}\right\}$ of the same length $l$ in $\operatorname{Circ}\left(n ;\left\{d_{1}, d_{2}, \cdots, d_{k}\right\}\right)$, the rotation distance $r(l)$ between $e_{1}$ and $e_{2}$ is $r(l)=\min \left\{r_{1}, r_{2}:\left(u_{1}+r_{1}\right)\left(u_{2}+r_{1}\right)=e_{2},\left(v_{1}+r_{2}\right)\left(v_{2}+r_{2}\right)=e_{1}\right\}$, where addition and difference are calculated inside $\mathbb{Z}_{n}$. Note that if $r(l)=l$, then the edges $e_{1}$ and $e_{2}$ are adjacent; if $r(l) \neq l$, then the edges $e_{1}$ and $e_{2}$ are non adjacent.

Throughout the paper we make use of the usual notation: $K_{n}$ for the complete graph on $n$ vertices, $K_{m, n}$ for the complete bipartite graph with independent sets of sizes $m$ and $n, P_{n}$ for the path on $n$ vertices, $C_{n}$ for the cycle on $n$ vertices, $D+F$ for the disjoint union $D \cup F$ of $D$ and $F, m F$ for $m$ disjoint copies of $F$ and $D \bigcup^{v} F$ for the union of $D$ and $F$ with a common vertex $v$ belongs to $F$ and $D$ Let $n_{1}, n_{2}, \cdots, n_{r}, r \geq 1$ be positive integers, $n_{1}, n_{r} \geq 1$ and $n_{i} \geq 0$ for $i \in\{2,3, \cdots, r-1\}$, the caterpillar $C_{r}\left(n_{1}, n_{2}, \cdots, n_{r}\right)$ is the tree obtained from the path $P_{r}:=x_{1} x_{2} \cdots x_{r}$ by joining vertex $x_{i}$ to $n_{i}$ new vertices, $i \in\{1,2, \cdots, r\}$. Other terminology not defined here can be found in [12].

\section{CODC of Circulant Graphs}

Consider the complete graph $K_{n}=\operatorname{Circ}(n ;\{1,2, \cdots,\lfloor n / 2\rfloor\})$. The authors of [13] introduced the notion of an 
orthogonal labelling. Given a graph $G=(V, E)$ with $n-1$ edges, a $1-1$ mapping $\psi: V \rightarrow \mathbb{Z}_{n}$ is an orthogonal labelling of $G$ if the following conditions are satisfied:

1) For every $l \in\{1,2, \cdots,[(n-1) / 2]\}, G$ contains exactly two edges of length $l$, and exactly one edge of length $(n / 2)$ if $n$ is even, and

2) For every $l \in\{1,2, \cdots,\lfloor(n-1) / 2\rfloor\}, r(l)=\{1,2, \cdots,\lfloor(n-1) / 2]\}$.

The following theorem of Gronau et al. [13] relates CODCs of $K_{n}$ and orthogonal labellings.

Theorem 2. ([13]) A CODC of $K_{n}$ by a graph $G$ exists if and only if there exists an orthogonal labelling of $G$.

Sampathkumar and Srinivasan [10], called an orthogonal $\{1,2, \cdots,\lfloor n / 2\rfloor\}$-labelling and generalized it to an orthogonal $\left\{d_{1}, d_{2}, \cdots, d_{k}\right\}$-labelling, where $\left\{d_{1}, d_{2}, \cdots, d_{k}\right\}$ is a sequence of positive integers with $1 \leq d_{1} \leq d_{2} \leq \cdots \leq d_{k} \leq\lfloor n / 2\rfloor$.

1) Either $n$ is odd or even and $d_{k} \neq n / 2$ :

Given a subgraph $G$ of $\operatorname{Circ}\left(n ;\left\{d_{1}, d_{2}, \cdots, d_{k}\right\}\right)$ with $2 k$ edges, a labelling of $G$, in $\mathbb{Z}_{n}$, is an orthogonal $\left\{d_{1}, d_{2}, \cdots, d_{k}\right\}$-labelling of $G$ if:

a) For every $l \in\left\{d_{1}, d_{2}, \cdots, d_{k}\right\}, G$ contains exactly two edges of length $l$, and

b) $r(l): l \in\left\{d_{1}, d_{2}, \cdots, d_{k}\right\}=\left\{d_{1}, d_{2}, \cdots, d_{k}\right\}$.

2) $n$ is even and $d_{k}=n / 2$

Given a subgraph $G$ of $\operatorname{Circ}\left(n ;\left\{d_{1}, d_{2}, \cdots, d_{k-1}, n / 2\right\}\right)$ with $2 k-1$ edges, a labelling of $G$, in $\mathbb{Z}_{n}$, is an orthogonal $\left\{d_{1}, d_{2}, \cdots, d_{k-1}, n / 2\right\}$-labelling of $G$ if:

a) For every $l \in\left\{d_{1}, d_{2}, \cdots, d_{k-1}\right\}, G$ contains exactly two edges of length $l$, and $G$ contains exactly one edges of length $(n / 2)$, and

b) $r(l): l \in\left\{d_{1}, d_{2}, \cdots, d_{k-1}\right\},=\left\{d_{1}, d_{2}, \cdots, d_{k-1}\right\}$, The following theorem of Sampathkumar and Simaringa [10], is a generalization of Theorem 2 .

Theorem 3 ([10]). A CODC of $\operatorname{Circ}\left(n ;\left\{d_{1}, d_{2}, \cdots, d_{k}\right\}\right)$ by a graph $G$ exists, if and only if there exists an orthogonal $\left\{d_{1}, d_{2}, \cdots, d_{k}\right\}$-labelling of $G$.

\section{CODCs of Circulant Graphs by Certain Graph Classes}

This section is devoted to constructing the cyclic orthogonal double covers (CODCs) of circulant graphs by different classes of graphs, complete bipartite graph as in Section 2.1, the union of the co-cycles graph with a star, the center vertex of which, belongs to the co-cycles graph as in Section 2.2 and graphs that are connected by a one vertex as in Section 2.3.

\subsection{CODCs by a Complete Bipartite Graph}

Theorem 4. For any positive integers $m, n, p$ such that $m p=n-1$, there exists a CODC of $(n-1)$-regular $\operatorname{Circ}(n ;\{1,2, \cdots,\lfloor n / 2]\})$ by $K_{m, p}$.

Proof Let us define $\psi: V\left(K_{m, p}\right) \rightarrow \mathbb{Z}_{n}$ by $\psi\left(v_{i+1}\right)=-i p$ for $0 \leq i \leq m-1$ and $\psi\left(v_{m+j+1}\right)=j+1$ for $0 \leq j \leq p-1$. Then the edges of length $l$ where

$l \in\{1,2, \cdots,\lfloor n / 2]\}=\left\{\psi\left(v_{i+1}\right), \psi\left(v_{m+l-i p}\right)\right\}$ and $\left\{\psi\left(v_{m-i}\right), \psi\left(v_{m-l+1+p(1+i)}\right)\right\}$, where $\psi\left(v_{i+1}\right)$ is defined for $i p+1 \leq l \leq(i+1) p$. For every $l \in\{1,2, \cdots,\lfloor n / 2\rfloor\}, \quad K_{m, p}$ contains exactly two edges of length $l$, and $\left\{r(l)=\psi\left(v_{m-i}\right)-\psi\left(v_{i+1}\right)-l: l \in\{1,2, \cdots,\lfloor n / 2\rfloor\}=\{1,2, \cdots,\lfloor n / 2\rfloor\}\right\}: l \in\{1,2, \cdots,\lfloor(n / 2)\rfloor\}=\{1,2, \cdots,\lfloor(n / 2)]\}$, and hence $K_{m, p}$ has an orthogonal labelling. By Theorem 3, there exists a CODC Of $(n-1)$-regular $\operatorname{Circ}(n ;\{1,2, \cdots,\lfloor n / 2\rfloor\})$ by $K_{m, p}$.

Let us define $m C_{l}^{a}$ to be the co-cycles graph (the union of $m$ cycles of length $l$ with a one vertex $a$ in common). In the following section we construct a CODCs of finite regular circulant graphs by $m C_{l}^{a} \bigcup^{a} K_{1, r}$ (the union of co-cycles graph with a star whose center vertex is the vertex $a$ ). 


\subsection{CODCs of Circulant Graph by $m C_{1}^{a} \cup^{a} K_{1, r}$}

Theorem 5 For any positive integer $n \geq 24$, there exists a CODC of $(n-5)$-regular $\operatorname{Circ}(n ;\{1,2, \cdots,\lfloor n / 2\rfloor\}) \backslash\{11,15\}$ by $4 C_{4}^{0} \bigcup^{0} K_{1, n-21}$.

Proof Let us define $\psi: V\left(4 C_{4}^{0} \cup^{0} K_{1, n-21}\right) \rightarrow \mathbb{Z}_{n}$ by $\psi\left(v_{i}\right)=i+8$ for $1 \leq i \leq n-17$, where,

$i \notin\{n-23, n-19\}, \psi\left(v_{n-16}\right)=0, \psi\left(v_{n-15}\right)=1, \psi\left(v_{n-14}\right)=2, \psi\left(v_{n-13}\right)=3, \psi\left(v_{n-12}\right)=5, \psi\left(v_{n-11}\right)=6$, $\psi\left(v_{n-10}\right)=7, \psi\left(v_{n-9}\right)=8, \psi\left(v_{n-8}\right)=n-7, \psi\left(v_{n-23}\right)=n-3$ and $\psi\left(v_{n-19}\right)=n-4$. Then the edges of length 1 are $\left\{\psi\left(v_{n-16}\right), \psi\left(v_{n-15}\right)\right\}=\{0,1\}$ and $\left\{\psi\left(v_{n-14}\right), \psi\left(v_{n-13}\right)\right\}=\{2,3\}$; those of length 2 are $\left\{\psi\left(v_{n-16}\right), \psi\left(v_{n-14}\right)\right\}=\{0,2\}$ and $\left\{\psi\left(v_{n-15}\right), \psi\left(v_{n-13}\right)\right\}=\{1,3\}$; those of length 3 are $\left\{\psi\left(v_{n-8}\right), \psi\left(v_{n-19}\right)\right\}=\{n-7, n-4\}$ and $\left\{\psi\left(v_{n-23}\right), \psi\left(v_{n-16}\right)\right\}=\{n-3,0\}$; those of length 4 are $\left\{\psi\left(v_{n-8}\right), \psi\left(v_{n-23}\right)\right\}=\{n-7, n-3\}$ and $\left\{\psi\left(v_{n-19}\right), \psi\left(v_{n-16}\right)\right\}=\{n-4,0\}$; those of length 5 are $\left\{\psi\left(v_{n-16}\right), \psi\left(v_{n-12}\right)\right\}=\{0,5\}$ and $\left\{\psi\left(v_{n-11}\right), \psi\left(v_{3}\right)\right\}=\{6,11\}$; those of length 6 are $\left\{\psi\left(v_{n-16}\right), \psi\left(v_{n-11}\right)\right\}=\{0,6\}$ and $\left\{\psi\left(v_{n-12}\right), \psi\left(v_{3}\right)\right\}=\{5,11\}$; those of length 7 are $\left\{\psi\left(v_{n-16}\right), \psi\left(v_{n-10}\right)\right\}=\{0,7\}$ and $\left\{\psi\left(v_{n-9}\right), \psi\left(v_{7}\right)\right\}=\{8,15\}$; those of length 8 are $\left\{\psi\left(v_{n-16}\right), \psi\left(v_{n-9}\right)\right\}=\{0,8\}$ and $\left\{\psi\left(v_{n-10}\right), \psi\left(v_{7}\right)\right\}=\{7,15\}$ the edges of length $l$ where $9 \leq l \leq\lfloor n / 2\rfloor\{11,15\}$ are $\left\{\psi\left(v_{n-16}\right), \psi\left(v_{l-8}\right)\right\}=\{0, l\}$ and $\left\{\psi\left(v_{n-(l+8)}\right), \psi\left(v_{n-16}\right)\right\}=\{n-l, 0\}$. For every $l \in\{1,2, \cdots,\lfloor n / 2]\}, 4 C_{4}^{0} \cup^{0} K_{1, n-21}$ contains exactly two edges of length $l$, and $\{r(l)=l+1: l \in\{1,3,5,7\}\},\{r(l)=l-1: l \in\{2,4,6,8\}\}$ and then $\{r(l)=\{1,2, \cdots,\lfloor n / 2\rfloor\}: l \in\{1,2, \cdots,\lfloor n / 2\rfloor\}\}$ and hence $4 C_{4}^{0} \cup^{0} K_{1, n-21}$ has an orthogonal labelling. By Theorem 3, there exists a CODC of $(n-5)$-regular $\operatorname{Circ}(n ;\{1,2, \cdots,\lfloor n / 2\rfloor\} \backslash\{11,15\})$ by $4 C_{4}^{0} \cup^{0} K_{1, n-21}$ for $n \geq 24$.

Theorem 6 For any positive integer $n \geq 5$, there exists a CODC of $(2 n-2)$-regular $\operatorname{Circ}(2 n ;\{1,2,3, \cdots, n-1\})$ by $2 C_{4}^{0} \bigcup^{0} K_{1,2 n-10}$.

Proof Let us define $\psi: V\left(2 C_{4}^{0} \cup^{0} K_{1,2 n-10}\right) \rightarrow \mathbb{Z}_{2 n}$ by $\psi\left(v_{i}\right)=i+2$ for $1 \leq i \leq 2 n-5$ where $i \neq n-3$, $\psi\left(v_{2 n-4}\right)=0, \psi\left(v_{2 n-3}\right)=1$ and $\psi\left(v_{n-3}\right)=2$. Then the edges of length 1 are $\left\{\psi\left(v_{2 n-4}\right), \psi\left(v_{2 n-3}\right)\right\}=\{0,1\}$ and $\left\{\psi\left(v_{2 n-3}\right), \psi\left(v_{n-3}\right)\right\}=\{1,2\}$; those of length 2 are $\left\{\psi\left(v_{n-4}\right), \psi\left(v_{n-2}\right)\right\}=\{n-2, n\}$ and $\left\{\psi\left(v_{n-2}\right), \psi\left(v_{n}\right)\right\}=\{n, n+2\}$; those of length $n-1$ are $\left\{\psi\left(v_{n-3}\right), \psi\left(v_{n-1}\right)\right\}=\{2, n+1\}$ and $\left\{\psi\left(v_{n-1}\right), \psi\left(v_{2 n-4}\right)\right\}=\{n+1,0\}$ the edges of length $l$ where $3 \leq l \leq n-2$ are $\left\{\psi\left(v_{2 n-4}\right), \psi\left(v_{l-2}\right)\right\}=\{0, l\}$ and $\left\{\psi\left(v_{2 n-(l+2)}\right), \psi\left(v_{2 n-4}\right)\right\}=\{-l, 0\}$. For every $l \in\{1,2,3, \cdots, n-1\}, \quad 2 C_{4}^{0} \cup^{0} K_{1,2 n-10}$ contains exactly two edges of length $l$, and since every two edges of the same length are adjacent then $\{r(l)=\{1,2,3, \cdots, n-1\}: l \in\{1,2,3, \cdots, n-1\}\}$ and hence $2 C_{4}^{0} \bigcup^{0} K_{1,2 n-10}$ has an orthogonal labelling. By Theorem 3, there exists a CODC of $(2 n-2)$-regular $\operatorname{Circ}(2 n ;\{1,2,3, \cdots, n-1\})$ by $2 C_{4}^{0} \bigcup^{0} K_{1,2 n-10}$ for $n \geq 5$.

Theorem 7 For any positive integer $n \geq 10$, there exists a CODC of $(n-1)$-regular $\operatorname{Circ}(n ;\{1,2, \cdots,\lfloor n / 2\rfloor\})$ by $2 C_{3}^{0} \bigcup^{0} K_{1, n-7}$.

Proof Let us define $\psi: V\left(2 C_{3}^{0} \cup^{0} K_{1, n-7}\right) \rightarrow \mathbb{Z}_{n}$ by $\psi\left(v_{i}\right)=i+3$ for $1 \leq i \leq n-7, \psi\left(v_{n-6}\right)=0$, $\psi\left(v_{n-5}\right)=1, \psi\left(v_{n-4}\right)=2, \psi\left(v_{n-3}\right)=n-3$ and $\psi\left(v_{n-2}\right)=n-2$. Then the edges of length 1 are $\left\{\psi\left(v_{n-6}\right), \psi\left(v_{n-5}\right)\right\}=\{0,1\}$ and $\left\{\psi\left(v_{n-5}\right), \psi\left(v_{n-4}\right)\right\}=\{1,2\}$; those of length 2 are 
$\left\{\psi\left(v_{n-6}\right), \psi\left(v_{n-4}\right)\right\}=\{0,2\}$ and $\left\{\psi\left(v_{n-2}\right), \psi\left(v_{n-6}\right)\right\}=\{n-2,0\}$; those of length 3 are

$\left\{\psi\left(v_{n-3}\right), \psi\left(v_{n-6}\right)\right\}=\{n-3,0\}$ and $\left\{\psi\left(v_{n-9}\right), \psi\left(v_{n-3}\right)\right\}=\{n-6, n-3\}$ the edges of length $l$ where

$4 \leq l \leq\lfloor n / 2\rfloor$ are $\left\{\psi\left(v_{n-6}\right), \psi\left(v_{l-3}\right)\right\}=\{0, l\}$ and $\left\{\psi\left(v_{n-(l+3)}\right), \psi\left(v_{n-6}\right)\right\}=\{n-l, 0\}$. For every

$l \in\{1,2, \cdots,\lfloor n / 2\rfloor\}, 2 C_{3}^{0} \cup^{0} K_{1, n-7}$ contains exactly two edges of length $l$, and since every two edges of the same length are adjacent then $\{r(l)=\{1,2, \cdots,\lfloor n / 2\rfloor\}: l \in\{1,2, \cdots,\lfloor n / 2\rfloor\}\}$ and hence $2 C_{3}^{0} \cup^{0} K_{1, n-7}$ has an orthogonal labelling. By Theorem 3, there exists a CODC of $(n-1)$-regular $\operatorname{Circ}(n ;\{1,2, \cdots,\lfloor n / 2\rfloor\})$ by $2 C_{3}^{0} \cup^{0} K_{1, n-7}$ for $n \geq 10$.

Theorem 8 For any positive integer $n \geq 13$, there exists a CODC of $(n-1)$-regular $\operatorname{Circ}(n ;\{1,2, \cdots,\lfloor(n / 2)]\})$ by $3 C_{3}^{0} \cup^{0} K_{1, n-10}$.

Proof Let us define $\psi: V\left(3 C_{3}^{0} \cup^{0} K_{1, n-10}\right) \rightarrow \mathbb{Z}_{n} \quad$ by $\psi\left(v_{i}\right)=i+4 \quad$ for $\quad 1 \leq i \leq n-9, \psi\left(v_{n-8}\right)=0$, $\psi\left(v_{n-7}\right)=1, \psi\left(v_{n-6}\right)=2, \psi\left(v_{n-5}\right)=n-3, \psi\left(v_{n-4}\right)=n-4$ and $\psi\left(v_{n-3}\right)=n-2$. Then the edges of length 1 are $\left\{\psi\left(v_{n-8}\right), \psi\left(v_{n-7}\right)\right\}=\{0,1\}$ and $\left\{\psi\left(v_{n-7}\right), \psi\left(v_{n-6}\right)\right\}=\{1,2\}$; those of length 2 are $\left\{\psi\left(v_{n-8}\right), \psi\left(v_{n-6}\right)\right\}=\{0,2\}$ and $\left\{\psi\left(v_{n-3}\right), \psi\left(v_{n-8}\right)\right\}=\{n-2,0\}$; those of length 3 are $\left\{\psi\left(v_{n-5}\right), \psi\left(v_{n-8}\right)\right\}=\{n-3,0\}$ and $\left\{\psi\left(v_{n-10}\right), \psi\left(v_{n-5}\right)\right\}=\{n-6, n-3\}$; those of length 4 are $\left\{\psi\left(v_{n-4}\right), \psi\left(v_{n-8}\right)\right\}=\{n-4,0\}$ and $\left\{\psi\left(v_{n-12}\right), \psi\left(v_{n-4}\right)\right\}=\{n-8, n-4\}$ the edges of length $l$ where $5 \leq l \leq\lfloor n / 2\rfloor$ are $\left\{\psi\left(v_{n-8}\right), \psi\left(v_{l-4}\right)\right\}=\{0, l\}$ and $\left\{\psi\left(v_{n-(l+4)}\right), \psi\left(v_{n-8}\right)\right\}=\{n-l, 0\}$. For every $l \in\{1,2, \cdots,\lfloor n / 2\rfloor\}, 3 C_{3}^{0} \cup^{0} K_{1, n-10}$ contains exactly two edges of length $l$, and since every two edges of the same length are adjacent then $\{r(l)=\{1,2, \cdots,\lfloor n / 2\rfloor\}: l \in\{1,2, \cdots,\lfloor n / 2\rfloor\}\}$ and hence $3 C_{3}^{0} \cup^{0} K_{1, n-10}$ has an orthogonal labelling. By Theorem 3, there exists a CODC of $(n-1)$-regular $\operatorname{Circ}(n ;\{1,2, \cdots,\lfloor n / 2\rfloor\})$ by $3 C_{3}^{0} \cup^{0} K_{1, n-10}$ for $n \geq 13$.

Theorem 9 For any positive integer $n \geq 17$, there exists a CODC of $(n-1)$-regular $\operatorname{Circ}(n ;\{1,2, \cdots,\lfloor(n / 2)]\})$ by $4 C_{3}^{0} \bigcup^{0} K_{1, n-13}$.

Proof Let us define $\psi: V\left(4 C_{3}^{0} \cup^{0} K_{1, n-13}\right) \rightarrow \mathbb{Z}_{n}$ by $\psi\left(v_{i}\right)=i+5$ for $1 \leq i \leq n-11$, $\psi\left(v_{n-10}\right)=0, \psi\left(v_{n-9}\right)=1, \psi\left(v_{n-8}\right)=2, \psi\left(v_{n-7}\right)=n-2, \psi\left(v_{n-6}\right)=n-3, \psi\left(v_{n-5}\right)=n-4$, and $\psi\left(v_{n-4}\right)=n-5$. Then the edges of length 1 are $\left\{\psi\left(v_{n-10}\right), \psi\left(v_{n-9}\right)\right\}=\{0,1\}$ and $\left\{\psi\left(v_{n-9}\right), \psi\left(v_{n-8}\right)\right\}=\{1,2 ;\}$; those of length 2 are $\left\{\psi\left(v_{n-10}\right), \psi\left(v_{n-8}\right)\right\}=\{0,2\}$ and $\left\{\psi\left(v_{n-7}\right), \psi\left(v_{n-10}\right)\right\}=\{n-2,0\}$; those of length 3 are $\left\{\psi\left(v_{n-6}\right), \psi\left(v_{n-10}\right)\right\}=\{n-3,0\}$ and $\left\{\psi\left(v_{n-11}\right), \psi\left(v_{n-6}\right)\right\}=\{n-6, n-3\}$; those of length 4 are $\left\{\psi\left(v_{n-5}\right), \psi\left(v_{n-10}\right)\right\}=\{n-4,0\}$ and $\left\{\psi\left(v_{n-13}\right), \psi\left(v_{n-5}\right)\right\}=\{n-8, n-4\}$; those of length 5 are $\left\{\psi\left(v_{n-4}\right), \psi\left(v_{n-10}\right)\right\}=\{n-5,0\}$ and $\left\{\psi\left(v_{n-15}\right), \psi\left(v_{n-4}\right)\right\}=\{n-10, n-5\}$ the edges of length $l$ where $6 \leq l \leq\lfloor n / 2\rfloor$ are $\left\{\psi\left(v_{n-10}\right), \psi\left(v_{l-5}\right)\right\}=\{0, l\}$ and $\left\{\psi\left(v_{n-(l+5)}\right), \psi\left(v_{n-10}\right)\right\}=\{n-l, 0\}$. For every $l \in\{1,2, \cdots,\lfloor n / 2\rfloor\}, 4 C_{3}^{0} \cup^{0} K_{1, n-13}$ contains exactly two edges of length $l$, and since every two edges of the same length are adjacent then $\{r(l)=\{1,2, \cdots,\lfloor n / 2\rfloor\}: l \in\{1,2, \cdots,\lfloor n / 2\rfloor\}\}$ and hence $4 C_{3}^{0} \cup^{0} K_{1, n-13}$ has an orthogonal labelling. By theorem 3, there exists a CODC of $(n-1)$-regular $\operatorname{Circ}(n ;\{1,2, \cdots,\lfloor n / 2\rfloor\})$ by 
$4 C_{3}^{0} \cup^{0} K_{1, n-13}$ for $n \geq 17$.

According to these results, we can pose the following conjecture:

Conjecture 1. For any positive integers $l, m, n$ such that $m<n$ and $l<n$, there exists a CODC of $(n-1)$-regular $\operatorname{Circ}(n ;\{1,2,3, \cdots,\lfloor n / 2\rfloor\})$ by $m C_{l}^{a} \bigcup^{a} K_{1, n-(1 m+1)}$.

\subsection{CODCs by $\left(D \bigcup^{a} F\right)$ Graphs that Are Connected by a One Vertex a}

Theorem 10 For any positive integer $n \geq 10$, there exists a CODC of $(n-1)$-regular $\operatorname{Circ}(n ;\{1,2, \cdots,\lfloor n / 2]\})$ by $K_{4} \cup^{4} K_{1, n-7}$.

Proof Let us define $\psi: V\left(K_{4} \cup^{4} K_{1, n-7}\right) \rightarrow \mathbb{Z}_{n}$ by $\psi\left(v_{i}\right)=i+8$ for $1 \leq i \leq n-9$, $\psi\left(v_{n-8}\right)=0, \psi\left(v_{n-7}\right)=1, \psi\left(v_{n-6}\right)=2, \psi\left(v_{n-5}\right)=4, \psi\left(v_{n-4}\right)=7$ and $\psi\left(v_{n-3}\right)=8$. Then the edges of length 1 are $\left\{\psi\left(v_{n-8}\right), \psi\left(v_{n-7}\right)\right\}=\{0,1\}$ and $\left\{\psi\left(v_{n-7}\right), \psi\left(v_{n-6}\right)\right\}=\{1,2\}$; those of length 2 are

$\left\{\psi\left(v_{n-6}\right), \psi\left(v_{n-5}\right)\right\}=\{2,4\}$ and $\left\{\psi\left(v_{n-8}\right), \psi\left(v_{n-6}\right)\right\}=\{0,2\}$; those of length 3 are

$\left\{\psi\left(v_{n-7}\right), \psi\left(v_{n-5}\right)\right\}=\{1,4\}$ and $\left\{\psi\left(v_{n-5}\right), \psi\left(v_{n-4}\right)\right\}=\{4,7\}$; those of length 4 are

$\left\{\psi\left(v_{n-8}\right), \psi\left(v_{n-5}\right)\right\}=\{0,4\}$ and $\left\{\psi\left(v_{n-5}\right), \psi\left(v_{n-3}\right)\right\}=\{4,8\}$; the edges of length $l$ where $5 \leq l \leq\lfloor n / 2\rfloor$ are $\left\{\psi\left(v_{n-5}\right), \psi\left(v_{l-4}\right)\right\}=\{4, l+4\} \quad$ and $\quad\left\{\psi\left(v_{n-(l+4)}\right), \psi\left(v_{n-5}\right)\right\}=\{n+4-l, 4\}$. For every $l \in\{1,2, \cdots,\lfloor n / 2]\}$, $K_{4} \cup^{4} K_{1, n-7}$ contains exactly two edges of length $l$, and since every two edges of the same length are adjacent then $\{r(l)=\{1,2, \cdots,\lfloor n / 2\rfloor\}: l \in\{1,2, \cdots,\lfloor n / 2\rfloor\}\}$ and hence $K_{4} \cup^{4} K_{1, n-7}$ has an orthogonal labelling. By Theorem 3, there exists a CODC of $(n-1)$-regular $\operatorname{Circ}(n ;\{1,2, \cdots,\lfloor n / 2\rfloor\})$ by $K_{4} \cup^{4} K_{1, n-7}$ for $n \geq 7$.

Theorem 11 For any prime number $n>11$, there exists a CODC of $(n-3)$-regular $\operatorname{Circ}(n ;\{1,2,3,5, \cdots,\lfloor n / 2\rfloor\})$ by $C_{5} \cup^{1} C_{3} \cup^{1} K_{1, n-11}$.

Proof Let us define $\psi: V\left(C_{5} \cup^{1} C_{3} \cup^{1} K_{1, n-11}\right) \rightarrow \mathbb{Z}_{n}$ by $\psi\left(v_{i}\right)=i+5$ for $1 \leq i \leq n-9$, $\psi\left(v_{n-8}\right)=0, \psi\left(v_{n-7}\right)=1, \psi\left(v_{n-6}\right)=n-1, \psi\left(v_{n-5}\right)=n-3$ and $\psi\left(v_{n-4}\right)=4$. Then the edges of length 1 are $\left\{\psi\left(v_{n-8}\right), \psi\left(v_{n-7}\right)\right\}=\{0,1\}$ and $\left\{\psi\left(v_{n-6}\right), \psi\left(v_{n-8}\right)\right\}=\{n-1,0\}$; those of length 2 are $\left\{\psi\left(v_{n-5}\right), \psi\left(v_{n-6}\right)\right\}=\{n-3, n-1\}$ and $\left\{\psi\left(v_{n-10}\right), \psi\left(v_{n-5}\right)\right\}=\{n-5, n-3\}$; those of length 3 are $\left\{\psi\left(v_{n-7}\right), \psi\left(v_{n-4}\right)\right\}=\{1,4\}$ and $\left\{\psi\left(v_{n-4}\right), \psi\left(v_{2}\right)\right\}=\{4,7\}$ the edges of length $l$ where $5 \leq l \leq\lfloor n / 2\rfloor$ are $\left\{\psi\left(v_{n-7}\right), \psi\left(v_{l-4}\right)\right\}=\{1, l+1\}$ and $\left\{\psi\left(v_{n-(l+4)}\right), \psi\left(v_{n-7}\right)\right\}=\{1-l, 1\}$. For every

$l \in\{1,2, \cdots,\lfloor n / 2\rfloor\}, C_{5} \cup^{1} C_{3} \cup^{1} K_{1, n-11}$ contains exactly two edges of length $l$, and since every two edges of the same length are adjacent then $\{r(l)=\{1,2,3,5, \cdots,\lfloor n / 2\rfloor\}: l \in\{1,2,3,5 m, \cdots,\lfloor n / 2\rfloor\}\}$ and hence

$C_{5} \cup^{1} C_{3} \cup^{1} K_{1, n-11}$ has an orthogonal labelling. By Theorem 3, there exists a CODC of $(n-3)$-regular $\operatorname{Circ}(n ;\{1,2,3,5, \cdots,\lfloor n / 2]\})$ by $C_{5} \cup^{1} C_{3} \cup^{1} K_{1, n-11}$ for $n>11$.

Theorem 12 For any positive integer $n \geq 1$, there exists a CODC of $(n-3)$-regular $\operatorname{Circ}(n ;\{1,2,3,5, \cdots,\lfloor n / 2\rfloor\})$ by $P_{5} \cup^{8} C_{3}(1,0, n-10)$.

Proof Let us define $\psi: V\left(P_{5} \cup^{8} C_{3}(1,0, n-10)\right) \rightarrow \mathbb{Z}_{n}$ by $\psi\left(v_{i}\right)=i+7$ for $1 \leq i \leq n-9, \psi\left(v_{n-8}\right)=0$, $\psi\left(v_{n-7}\right)=1, \psi\left(v_{n-6}\right)=2, \psi\left(v_{n-5}\right)=4, \psi\left(v_{n-4}\right)=6, \psi\left(v_{n-3}\right)=5$ and $\psi\left(v_{n-2}\right)=3$. Then the edges of length 1 are $\left\{\psi\left(v_{n-7}\right), \psi\left(v_{n-6}\right)\right\}=\{1,2\}$ and $\left\{\psi\left(v_{n-8}\right), \psi\left(v_{n-7}\right)\right\}=\{0,1\}$; those of length 2 are $\left\{\psi\left(v_{n-5}\right), \psi\left(v_{n-4}\right)\right\}=\{4,6\}$ and $\left\{\psi\left(v_{n-4}\right), \psi\left(v_{1}\right)\right\}=\{6,8\}$; those of length 3 are $\left\{\psi\left(v_{n-3}\right), \psi\left(v_{1}\right)\right\}=\{5,8\}$ 
and $\left\{\psi\left(v_{n-6}\right), \psi\left(v_{n-3}\right)\right\}=\{2,5\}$ the edges of length $l$ where $5 \leq l \leq\lfloor n / 2\rfloor$ are $\left\{\psi\left(v_{n-2}\right), \psi\left(v_{l-4}\right)\right\}=\{3, l+3\}$ and $\left\{\psi\left(v_{n-(l+4)}\right), \psi\left(v_{n-2}\right)\right\}=\{3-l, 3\}$. For every $l \in\{1,2,3,5, \cdots,\lfloor n / 2\rfloor\}, P_{5} \cup^{8} C_{3}(1,0, n-10)$ contains exactly two edges of length $l$, and since every two edges of the same length are adjacent then $\{r(l)=\{1,2,3,5, \cdots,\lfloor n / 2\rfloor\}: l \in\{1,2,3,5 m, \cdots,\lfloor n / 2\rfloor\}\}$ and hence $P_{5} \cup^{8} C_{3}(1,0, n-10)$ has an orthogonal labelling. By Theorem 3, there exists a CODC of $(n-3)$-regular $\operatorname{Circ}(n ;\{1,2,3,5, \cdots,\lfloor n / 2\rfloor\})$ by $P_{5} \cup^{8} C_{3}(1,0, n-10)$ for $n \geq 11$.

Conjecture 2. For any positive integers $m, n$ so that $m<n$, there exists a CODC of $(n-1)$-regular $\operatorname{Circ}(n ;\{1,2,3, \cdots,\lfloor n / 2\rfloor\})$ by $K_{m} \cup^{a} K_{1, \frac{2 n-(m(m-1)+2)}{2}}$.

\section{General CODCs of Circulant Graph}

In constructing CODCs a natural approach is to try to use given CODCs to obtain CODCs of a larger Circulant Graph. That is we will do in the following theorem.

Theorem 13 For any positive integers $m, n$, if there exists a CODC of $\operatorname{Circ}\left(n ;\left\{d_{1}, d_{2}, \cdots, d_{k}\right\}\right)$ by $G$ with respect to $\mathbb{Z}_{n}$. Then there exists a CODC of $\operatorname{Circ}\left(m n ;\left\{e_{1}, e_{2}, \cdots, e_{r}\right\}\right)$ where $1 \leq e_{1} \leq e_{2} \leq \cdots \leq e_{r} \leq\lfloor(m n) / 2\rfloor$ by $G_{1}$ with respect to $\mathbb{Z}_{m n}$.

Proof Let the $\operatorname{Circ}\left(n ;\left\{d_{1}, d_{2}, \cdots, d_{k}\right\}\right)$ has a CODC by $G$ with respect to $\mathbb{Z}_{n}$. Then the graph $G$ has an orthogonal $\left\{d_{1}, d_{2}, \cdots, d_{k}\right\}$-labelling with respect to $\mathbb{Z}_{n}$. And hence, for every $d_{i} \in\left\{d_{1}, d_{2}, \cdots, d_{k}\right\}$, $G$ contain exactly two edges of the length $d_{i}$ as $\left\{v_{d_{i}}, v_{d_{i}}+d_{i}\right\}$ and $\left\{v_{-d_{i}}, v_{-d_{i}}-d_{i}\right\}$ where $i \in\{1,2, \cdots, k\}$ and $v_{d_{i}}, v_{-d_{i}} \in \mathbb{Z}_{n}$. And $r\left(d_{i}\right): d_{i} \in\left\{d_{1}, d_{2}, \cdots, d_{k}\right\}=\left\{d_{1}, d_{2}, \cdots, d_{k}\right\}$ to construct a CODC of

$\operatorname{Circ}\left(m n ;\left\{e_{1}, e_{2}, \cdots, e_{r}\right\}\right)$ for $r \in\{1,2, \cdots,\lfloor m n / 2\rfloor\}$ by $G_{1}$ with respect to $\mathbb{Z}_{m n}$, the graph $G_{1}$ must have an orthogonal $\left\{e_{1}, e_{2}, \cdots, e_{r}\right\}$-labelling with respect to $\mathbb{Z}_{m n}$. From the orthogonal labelling of $G$ we can obtain an orthogonal labelling of $G_{1}$ as follows

Case 1: Either $n$ is odd or even and $d_{k} \neq n / 2$

$$
e_{r}=e_{\alpha(2 k+1)+j}= \begin{cases}d_{j+\alpha n}, & \text { if } 1 \leq j \leq k \\ (\alpha+1) n-d_{2 k+1-j}, & \text { if } K+1 \leq j \leq 2 k \\ \alpha n, & \text { if } j=0 \text { and } \alpha \neq 0\end{cases}
$$

where $0 \leq \alpha \leq m-1$. For every $l \in\left\{e_{1}, e_{2}, \cdots, e_{r}\right\}, \quad G_{1}$ contain exactly two edges of the length $l$ as $\left\{v_{e_{r}}, v_{e_{r}}+e_{r}\right\}$ and $\left\{v_{-e_{r}}, v_{-e_{r}}-e_{r}\right\}$ where

and

$$
v_{e_{r}}= \begin{cases}v_{d_{j}}, & \text { if } 1 \leq j \leq k \\ v_{-\left(d_{2 k+1-j}\right)}, & \text { if } K+1 \leq j \leq 2 k \\ \beta, & \text { if } j=0 \text { and } \alpha \neq 0: \beta \in \mathbb{Z}_{m n}\end{cases}
$$

$$
v_{-e_{r}}= \begin{cases}v_{-d_{j}}, & \text { if } 1 \leq j \leq k \\ v_{\left(d_{2 k+1-j}\right)}, & \text { if } K+1 \leq j \leq 2 k \\ \beta, & \text { if } j=0 \text { and } \alpha \neq 0 .\end{cases}
$$

By the definition of $e_{r}, v_{e_{r}}$ and $v_{-e_{r}}$, we have $r(l): l \in\left\{e_{1}, e_{2}, \cdots, e_{r}\right\}=\left\{e_{1}, e_{2}, \cdots, e_{r}\right\}$. Then the graph $G_{1}$ has an orthogonal $\left\{e_{1}, e_{2}, \cdots, e_{r}\right\}$-labelling with respect to $\mathbb{Z}_{m n}$. 
Case 2: $n$ is even and $d_{k}=n / 2$

$$
e_{r}=e_{2 \alpha k+j}= \begin{cases}d_{j+\alpha n}, & \text { if } 1 \leq j \leq k \\ n-d_{2 k-j}+\alpha n, & \text { if } K+1 \leq j \leq 2 k-1 \\ \alpha n, & \text { if } j=0 \text { and } \alpha \neq 0\end{cases}
$$

where $0 \leq \alpha \leq m-1$. For every $l \in\left\{e_{1}, e_{2}, \cdots, e_{r}\right\}, G_{1}$ contain exactly two edges of the length $l$ as $\left\{v_{e_{r}}, v_{e_{r}}+e_{r}\right\}$ and $\left\{v_{-e_{r}}, v_{-e_{r}}-e_{r}\right\}$ where

$$
v_{e_{r}}= \begin{cases}v_{d_{j}}, & \text { if } 1 \leq j \leq k \\ v_{-\left(d_{2 k-j}\right)}, & \text { if } K+1 \leq j \leq 2 k-1 \\ \beta, & \text { if } j=0 \text { and } \alpha \neq 0: \beta \in \mathbb{Z}_{m n}\end{cases}
$$

and

$$
v_{-e_{r}}= \begin{cases}v_{-d_{j}}, & \text { if } 1 \leq j \leq k \\ v_{d_{2 k-j}}, & \text { if } K+1 \leq j \leq 2 k \\ \beta, & \text { if } j=0 \text { and } \alpha \neq 0 .\end{cases}
$$

By the definition of, $e_{r}, v_{e_{r}}$ and $v_{-e_{r}}$, we have $r(l): l \in\left\{e_{1}, e_{2}, \cdots, e_{r}\right\}=\left\{e_{1}, e_{2}, \cdots, e_{r}\right\}$. Then the graph $G_{1}$ has an orthogonal $\left\{e_{1}, e_{2}, \cdots, e_{r}\right\}$-labelling with respect to $\mathbb{Z}_{m n}$. By Theorem 3, there exists a CODC of $\operatorname{Circ}\left(m n ;\left\{e_{1}, e_{2}, \cdots, e_{r}\right\}\right)$ by $G_{1}$ with respect to $\mathbb{Z}_{m n}$.

\section{Conclusion}

In this paper we are concerned with the orthogonal labelling of CODCs of finite regular circulant graphs. We constructed CODCs by certain classes of graphs such as complete bipartite graph, the union of the co-cycles graph with a star, the center vertex of which belongs to the co-cycles graph and graphs that are connected by a one vertex. Finally we introduced general CODCs of the circulant graph.

\section{References}

[1] Gronau, H.-D.O.F., Hartmann, S., Grüttmüller, M., Leck, U. and Leck, V. (2002) On Orthogonal Double Covers of Graphs. Design Codes Cryptography, 27, 49-91. http://dx.doi.org/10.1023/A:1016546402248

[2] El-Shanawany, R. and Higazy, M. (2008) Orthogonal Double Covers of Complete Graphs by Certain Spanning Subgraphs. Australasian Journal of Combinatorics, 42, 223-228.

[3] El Shanawany, R., Higazy, M. and Scapellato, R. (2010) A Note on Orthogonal Double Covers of Complete Bipartite Graphs by a Special Class of Six Caterpillars. AKCE International Journal of Graphs and Combinatorics, 7, 1-4.

[4] El-Shanawany, R.A., Higazy, M.S. and Scapellato, R. (2009) Orthogonal Double Covers of Complete Bipartite Graphs by the Union of a Cycle and a Star. Australasian Journal of Combinatorics, 43, 281-293.

[5] El-Shanawany, R. and Higazy, M. (2007) General Symmetric Starter of Orthogonal Double Covers of Complete Bipartite Graph. International Journal of Mathematics and Mathematical Sciences, 2007, Article ID: 42892. http://dx.doi.org/10.1155/2007/42892

[6] El Shanawany, R., Higazy, M. and El Mesady, A. (2013) On Cartesian Products of Orthogonal Double Covers. International Journal of Mathematics and Mathematical Sciences, 2013, Article ID: 265136.

[7] Scapellato, R., El-Shanawany, R. and Higazy, M. (2009) Orthogonal Double Covers of Cayley Graphs. Discrete Applied Mathematics, 157, 3111-3118. http://dx.doi.org/10.1016/j.dam.2009.06.005

[8] Hartmann, S. and Schumacher, U. (2004) Orthogonal Double Covers of General Graphs. Discrete Applied Mathematics, 138, 107-116. http://dx.doi.org/10.1016/S0166-218X(03)00274-9

[9] Sampathkumar, R. and Sriram, V. (2008) Orthogonal $\sigma$-Labelling of Graphs. AKCE International Journal of Graphs and Combinatorics, 5, 57-60.

[10] Sampathkumar, R. and Srinivasan, S. (2011) Cyclic Orthogonal Double Covers of 4-Regular Circulant Graphs. Discrete Mathematics, 311, 2417-2422. http://dx.doi.org/10.1016/j.disc.2011.06.021 
[11] El Shanawany, R., and Shabana, H. (2014) On Orthogonal Double Covers of Circulant Graphs. British Journal of Mathematics \& Computer Science, 4, 394-401.

[12] Balakrishnan, R. and Ranganathan, K. (2012) A Textbook of Graph Theory. Springer, Berlin. http://dx.doi.org/10.1007/978-1-4614-4529-6

[13] Gronau, H.-D.O.F., Mullin, R.C. and Rosa, A. (1997) On Orthogonal Double Covers of Complete Graphs by Trees. Graphs and Combinatorics, 13, 251-262. 